\title{
The SPM kinematic catalogue of Galactic planetary nebulae
}

\author{
Jose-Alberto López ${ }^{1}$, Michael G. Richer ${ }^{1}$, Maria-Teresa García-Díaz ${ }^{1}$, \\ David M. Clark ${ }^{1}$, John Meaburn ${ }^{2}$, Hortensia Riesgo ${ }^{1}$, \\ Wolfgang Steffen ${ }^{1}$ and Myfanwy Lloyd ${ }^{2}$ \\ ${ }^{1}$ Instituto de Astronomía, Universidad Nacional Autónoma de México, \\ Campus Ensenada, C.P. 22800, Baja California, México \\ email: jal, richer, tere, dmclark, hriesgo, wsteffen@astrosen.unam.mx \\ ${ }^{2}$ Jodrell Bank Center for Astrophysics, School of Physics and Astronomy \\ University of Manchester, M 13 9PL, UK \\ email: meaburjr@yahoo.co.uk, Myfanwy.Lloyd@manchester.ac.uk
}

\begin{abstract}
The San Pedro Mártir kinematic catalogue of galactic planetary nebulae provides spatially resolved, long-slit, Echelle spectra for about 600 planetary nebulae, representing 55 observing runs and about 4000 individual integrations to date in this first release. The project is ongoing and will continue adding spectra to the database. The data are presented wavelength calibrated and corrected for heliocentric motion. This is the most extensive and homogeneous single source of data concerning the internal kinematics of the ionized nebular material in planetary nebulae. The catalogue is available through the world wide web at http://kincatpn.astrosen.unam.mx and an article will a full description of the catalogue will soon appear in the RevMexAA.
\end{abstract}

Keywords. Planetary nebulae: general, catalogs

\section{Introduction}

The kinematics of the ionized nebular shell of planetary nebulae (PNe) provides key information on the physics that drives their expansion and which is necessary to understand their formation and evolution, as well as their role as galactic chemical contaminants of processed material through stellar mass-loss from the TP-AGB to the white dwarf stages. $\mathrm{PNe}$ are excellent tracers of galactic structure in our own and other galaxies and have been used as extra-galactic standard candles from the distribution of their [O III] luminosity function, which depends to a certain degree on the evolution of the ionized nebular shell. The complex morphological structures of PNe revealed in recent times by the HST and ground-based telescopes that achieve subarcsec image quality demand the knowledge of detailed expansion patterns to disentangle real outflows from projection, scattering and light-cone effects, particularly in the early stages of the ionized, forming nebular shells and to identify differences in expansion patterns among different morphological classes and isolate components from different mass-loss episodes and age of the nebular shell. Furthermore, the growing high-quality data of the last decade on PNe has made clear the relevance of embedded collimated outflows, poly-polarity and other complex symmetries in their structural development. Therefore, to understand the dynamics of PNe from an in-depth perspective a systematic and homogeneous set of high quality, spatially resolved, kinematic information of high spectral resolution, spanning most of the evolutionary stages, morphologies, progenitor masses and galactic populations is required. That is what the SPM kinematic catalogue on galactic planetary nebulae aims to provide. 


\section{The data}

The data for this first release of the SPM catalogue have been obtained over 55 observing runs. Fifty two observing runs were obtained at the $2.1 \mathrm{~m}, \mathrm{f} / 7.5$ telescope in San Pedro Mártir National Observatory, México, with the Manchester Echelle Spectrometer (Meaburn et al. 2003) and three additional runs in the southern hemisphere at the $3.9 \mathrm{~m}, \mathrm{f} / 8$, Anglo-Australian Telescope, two of them using a twin MES and a third one with the UCLES spectrometer (Diego et al. 1990) used in single order format.

MES is an Echelle, long-slit spectrometer optimized for nebular work. MES does not have a cross disperser, it isolates single orders using interference filters. For example, the $87^{\text {th }}$ order covers the $\mathrm{H} \alpha$, He II $\lambda 6560 \AA$, C II $\lambda 6578 \AA$, and the [N II] $\lambda \lambda 6548$, $6583 \AA$ emission lines, and the $114^{\text {th }}$ order contains the [O III] $\lambda 5007 \AA$ line. The order containing the $[\mathrm{S} \mathrm{II}] \lambda \lambda 6717,6731 \AA$ emission lines has also been observed for some objects.

All of the spectra are contained in a database accessible via the world wide web at http://kincat.astrosen.unam.mx/. After selecting an individual PN an image of the nebula with accurate slit positions overlaid on it is presented and all the available data for that PN is displayed in a table below the image, see Figure 1. The table provides at the top the common name and PNG identifier and the run(s) identifier for when it was observed. The data consists of "Full" meaning the full spectral range (see Figure 2), calibrated in wavelength or individual bi-dimensional line spectra labeled by the

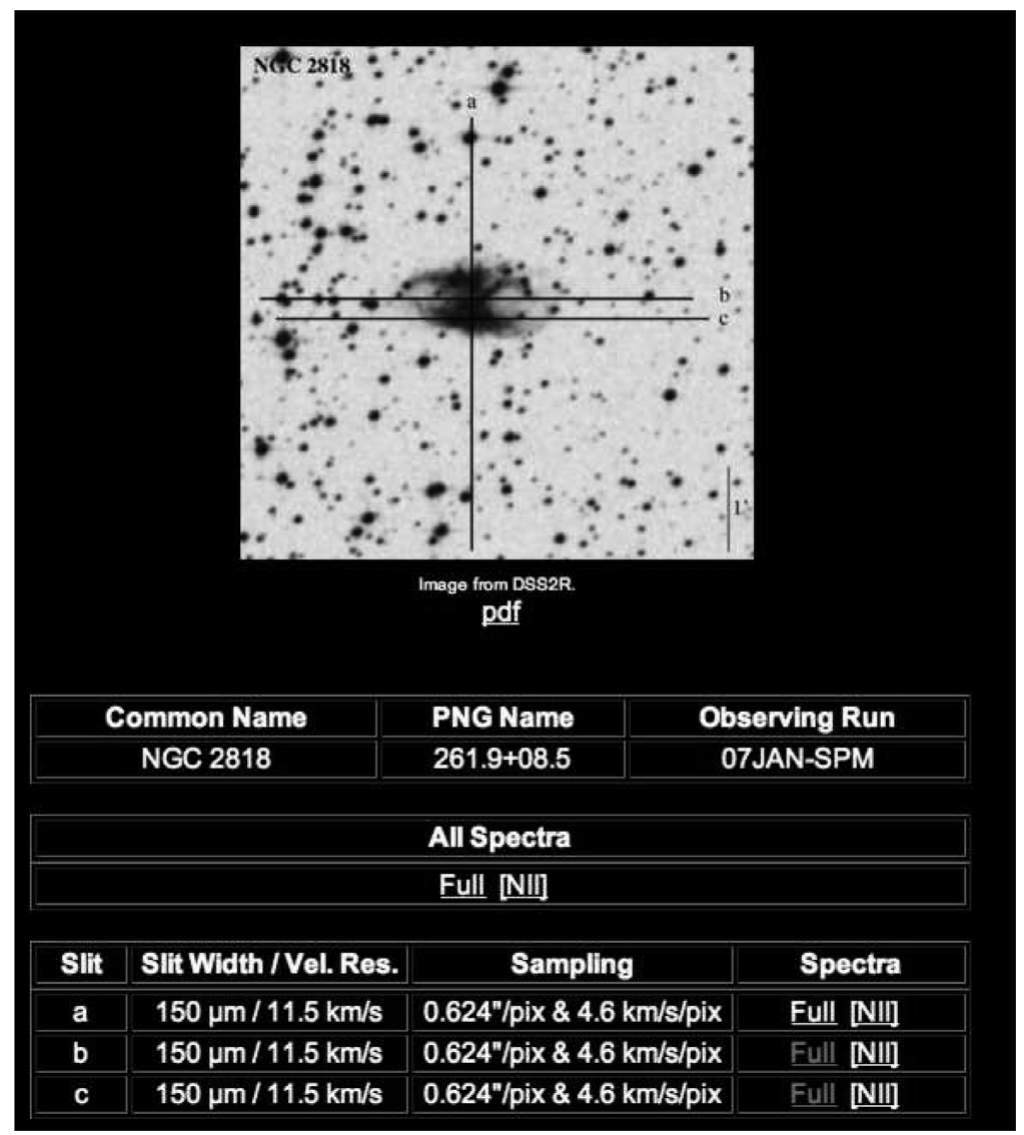

Figure 1. Example of the presentation of a PN in the catalogue. 
corresponding line identifier such as $[\mathrm{N}$ II] that refers to the $\lambda 6583 \AA$ emission line (see Figure 3) or [O III] referring to the $\lambda 5007 \AA$ emission line. In the latter case this is the only line of interest in the order, therefore this is never labeled as "Full" since it is always presented as an individual line spectrum. All individual line spectra are presented calibrated to heliocentric velocity.

Next in the table and below the label "All Spectra" there are underlined keywords such as Full, $\mathrm{H} \alpha+[\mathrm{N} \mathrm{II}]$, [O III] or [N II], in case that the object has multiple observations. Clicking on any of these keywords all available data for the corresponding keyword for that object will be displayed to the right of the table. Below each image or spectrum there is a link for downloading a pdf file of it. Wavelength calibrated FITS files for individual objects can also be requested through the contact email address that appears in the front page of the web based catalogue. The full slit length in MES is in most cases about 5 arcmin. This length is dependent on the CCD size format so it varies slightly in some cases depending on the CCD we used at the time. For the CCDs we used throughout these runs the slit varies from 5 to 7 arcmin. Only in the case of the spectra obtained at the AAT with UCLES the slit length is less than one arcmin, specifically 57 arcsec. In many cases it is not necessary to depict the full length of the slit on an image, only the portion that produces relevant data. All the images that accompany each object and show the location of the slits on them are oriented with north up and east left and show an angular scale bar. Likewise, all the position-velocity or bi-dimensional line profiles for each object are presented with an angular scale along the slit axis. Given the relatively large slit length most of the images are drawn either from the Digital Sky Survey or have been obtained with MES in its imaging mode, for only a few cases they are from other sources. A legend at the bottom of the image with the slits shows the image origin.

The slit width has been for most objects $150 \mu \mathrm{m} \equiv 11.5 \mathrm{~km} \mathrm{~s}^{-1}$ in velocity resolution, for some bright objects we have used the $70 \mu \mathrm{m} \equiv 6 \mathrm{~km} \mathrm{~s}^{-1}$ and for some faint targets in some cases we have used a $300 \mu \mathrm{m} \equiv 22 \mathrm{~km} \mathrm{~s}^{-1}$. Since on some occasions we observed a target in more than one run, possibly with a different CCD or with different slit widths, or different on-chip binning, for each object we list in the associated table for that PN all its associated slit positions, the slit width, its equivalent velocity resolution and associated spectral and spatial sampling information. Clicking on the keywords of the last column in the table, the individual "Full" or line spectra are displayed.

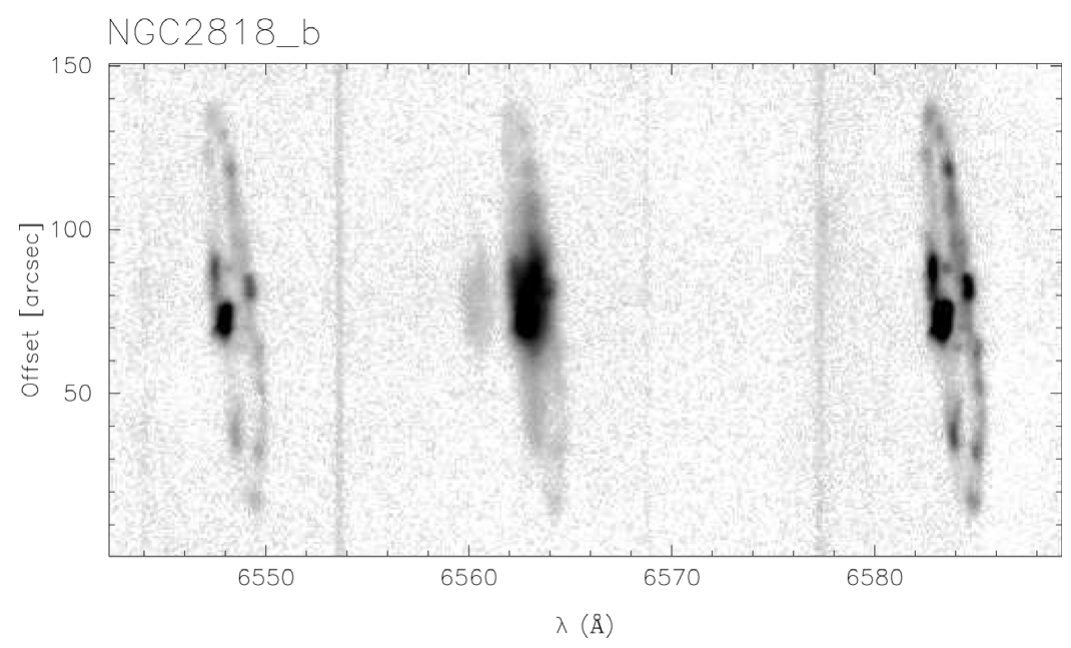

Figure 2. Full spectral range for the $\mathrm{H}_{\alpha}$ order corresponding to slit b in NGC 2818. 


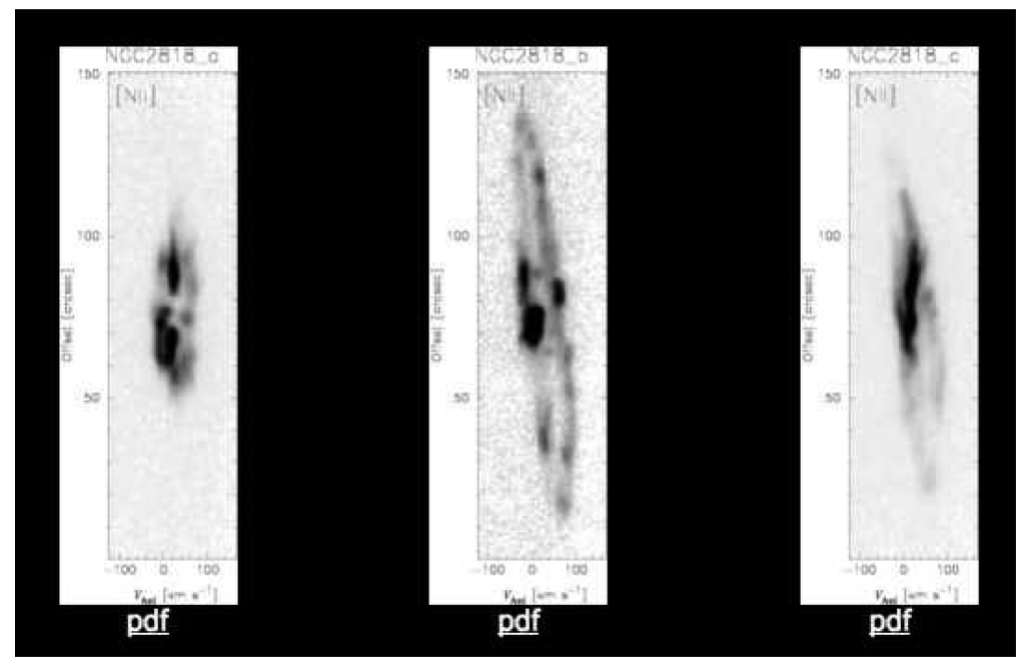

Figure 3. Individual [N II] P-V arrays from the 3 slit positions indicated in Fig. 1 for NGC 2818.

\section{The database}

To connect to the database the user must provide her or his email address. After logging in the search page. The database provides the option to search for an individual PN by its common name or PNG name, following the IAU format. For the common name the prefix PN used by SIMBAD is excluded and for the PNG name the prefix PN G used by SIMBAD is also excluded.

In addition to searching for an individual object the entire database can be browsed with the option Full List. The list can be ordered by common or PN G name. Objects can be searched and grouped by galactic coordinates or within a range of them. Additional, extended search methods are listed that exploit the small wavelength range covered by the Echelle orders. The ultimate objective is that this catalogue be an effective tool in helping us to better understand the formation and evolution of planetary nebulae. This work is a companion to the SPM planetary nebulae kinematic catalogue of extragalactic planetary nebulae (Richer et al. 2010) also available on line through one of the links located at the end of the search page. An article with a full description of the catalogue will soon appear in the RevMexAA.

The authors gratefully acknowledge the numerous people that have provided valuable resources and contributed their time and talents to make this project possible.The participating members in this project, including students and postdocs, have benefited throughout the years from the generous financial support of DGAPA-UNAM through several PAPIIT grants as well as several CONACYT grants.

\section{References}

Diego, F., Charalambous, A., Fish, A. C., \& Walker, D. 1990, Proc. SPIE, on Instrumentation in Astronomy VII, Vol. 1235, 562

Meaburn, J., López, J. A., Gutiérrez, L., Quiróz, F., Murillo, J. M., Valdéz, J., \& Pedrayes, M. H. 2003, Revista Mexicana de Astronomía y Astrofísica, 39, 185

Richer, M. G., López, J. A., Díaz-Méndez, E., Riesgo, H., Báez, S.-H., García-Díaz, M. T., Meaburn, J., Clark, D. M., Calderón Olvera, R. M., López Soto, G., \& Toledano Rebolo, O. 2010, Revista Mexicana de Astronomía y Astrofísica, 46, 19 